\title{
Evaluation of 100 Cases of Mortality after Cardiac Surgery: A Single-Center Experience in Bangladesh
}

\author{
Dharmendra Joshi ${ }^{1}$, Mayank Acharya ${ }^{1}$, Niraj Bhattarai ${ }^{1}$, Md. Abir Tazim Chowdhury ${ }^{1}$, \\ Md. Alauddin ${ }^{1}$, and Md. Rezwanul Hoque ${ }^{1}$ \\ ${ }^{1}$ Bangabandhu Sheikh Mujib Medical University
}

July 21, 2020

Abstract

Background: Vigilance ensures safety in cardiac surgery. Performance in cardiac surgery is often measured by short-term mortality. Several risk factors like advanced age, female gender, higher body mass index, decreased left ventricular function, emergent, and redo operations have appeared recurrently as poor prognostic variables. Evaluation of postoperative mortality is crucial to find loopholes to provide proper care and reduce preventable mortality after cardiac surgery in developing countries with limited infrastructures and resources.

Methods: This is a retrospective study conducted in the Department of Cardiac Surgery, Bangabandhu Sheikh Mujib Medical University. Perioperative data of 100 cases of mortality after cardiac surgery performed from 1 January 2014 to 30 May 2018 were collected from the university medical record. The data on age, gender, body mass index, preoperative investigations, diagnoses, types of operations, details of cardiopulmonary bypass, and postoperative period of the study populations were evaluated.

Results: During the study period, about 1627 cases of cardiac surgery were done with an overall mortality rate of $6.15 \%$. The mean age was $41.05 \pm 20.19$ ( 0 - 68) years, and $66 \%$ of patients were male. Preoperative ejection fraction (EF) of the study population was an average $56.63 \% \pm 11.85 \% ; 9 \%$ of the patients had $\mathrm{EF}<40 \%$. Off-pump coronary artery bypass $(32.27 \%)$ was the most commonly performed surgery followed by mitral valve replacement (24.28\%). On-pump cardiac surgery was done among $65 \%$ of the study population with a mean cross-clamp time and bypass time of $32.56 \pm 11.55$ minutes and $80.57 \pm 18.09$ minutes, respectively. Most of the mortality was found in the first two weeks after surgery.

Conclusion: Mortality after cardiac surgery is multifactorial. A large-scale prospective study with comparative groups is required to find out preventable measures of mortality after cardiac surgery which will improve the quality of services provided to the patients in developing countries.

\section{Keywords:}

Cardiac Surgery, Morbidity, Mortality, Evaluation

\section{Introduction}

Vigilance ensures safety in cardiac surgery but despite all improved technologies and infrastructures for perioperative support of cardiac surgery, mortality is inevitable. Mortality is an unequivocal and easy method to evaluate outcomes after cardiac surgery. So, this parameter is widely used to assess the outcome after cardiac surgery. But different schools of thought advocate the optimal period to assess mortality after cardiac surgery viz. some prefer for in-hospital mortality, 30 days mortality, or even mortality after 60 days up to 6 months after cardiac surgery [1]. Performance in cardiac surgery is often measured by short-term mortality. 
Several risk factors like advanced age, female gender, higher body mass index, decreased left ventricular function, emergent and redo operations have appeared recurrently as poor prognostic variables [2] [3] [4].

Consideration of the risks and prediction of outcomes after cardiac surgery based on the preoperative analysis of the clinical information and investigations of the patients help to assess, evaluate and focus for a better outcome and improved quality of care for the patients undergoing cardiac surgery [5] [6]. But there are continuous changes in the patient characteristics and surgical methods. Like octogenarian with more advanced and diffuse coronary artery disease, left ventricular dysfunction, failed previous angioplasty, more serious comorbidities, and multiple redo cases are referred for coronary artery bypass surgery (CABG). Similarly, the complex preoperative profile of patients undergoing other cardiac surgery demands periodic updates of the perioperative predictors [7].

Many kinds of researches and advancements have been done for identifying and defining risk factors for predicting outcomes after CABG. However, valve surgery has lagged behind that for CABG which may be due to fewer valve surgeries performed than CABG, thus demanding more time to acquire adequate cases for outcome evaluation. Also, the changes in patient characteristics, valve location, surgical procedures, and outcomes over time affect the mortality rate and risk factors [6]. So, we are auditing all the postoperative in-hospital mortality cases who underwent various cardiac surgery in our department in a specified period to evaluate the fields of improvement for a better outcome after cardiac surgery.

\section{Materials and Methods}

A retrospective study was conducted in the Department of Cardiac Surgery, Bangabandhu Sheikh Mujib Medical University collecting data from the medical record section of the university. Proper written consent for the study has been taken from the university. The data of 100 cases of mortality after cardiac surgery from 1 January 2014 to 30 May 2018 was evaluated by collecting the data on age, gender, body mass index, preoperative investigations like blood tests, electrocardiogram (ECG), echocardiography, and spirometry. Similarly, preoperative diagnoses, types of operations, details of cardiopulmonary bypass, and postoperative period of the study populations were extracted and evaluated.

Nashef et al. defined early or operative death or mortality as any of the following: 1) death in the same hospital as the operation took place, before discharge from hospital; 2) death in the same hospital or at another hospital but before discharge from hospital; 3) death within 30 days of surgery regardless of location; 4) death within 90 days of surgery regardless of location; 5) a compound of some or all of the above [8].

Analysis of data was done using Statistical Package for Social Science (SPSS) version 24.0 for windows software. Categorical variables are reported as numbers (percent), whereas continuous variables are expressed as mean \pm standard deviation, and range is shown whenever feasible. No groups were compared in this study. Hence, the study is limited with simple statistical analysis and no advanced statistic was applicable. The statistical analysis was done to find out the relation between the variables and the mortality and generate the information that could be utilized for better management and save more patients in the future.

\section{Results}

During the study period, about 1627 cases of cardiac surgery were done in our medical university stating an overall mortality rate of $6.15 \%$ which is a little higher. It may be because our university being a tertiary referral center, many complicated cases are operated with limited resources and infrastructure. But the overall mortality rate is improving every year with the advancement in skill and support systems like transesophageal echocardiography (TEE), sustained low-efficiency dialysis (SLED), and stringent intermittent pneumatic compression (IPC), declining the mortality rate below $5 \%$ in 2020. The baseline characteristics of the study population are shown in Table 1 . The mean age was $41.05 \pm 20.19(0-68)$ years, and $66 \%$ of patients were male. Most of the study population (54\%) had a normal body mass index (BMI) with an average BMI of $22.67 \pm 8.66 \mathrm{~kg} / \mathrm{m}^{2}$ and range $10.77-35.92 \mathrm{~kg} / \mathrm{m}^{2}$. The previous history of the patients revealed hypertension in $35 \%$, diabetes mellitus in $36 \%$, and ischemic heart disease/myocardial infarction in $36 \%$ of the study population. 
Preoperative investigations were evaluated. A higher level of plasma Red Cell Distribution Width (RDW) level was found associated with the mortality of the patients. Similarly, an increased C-reactive protein (CRP) level $(7.74 \pm 16.55)$ was also found in the study, more in men than women $(\mathrm{p}<0.03)$ (TableS1, Supplementary data). The echocardiography showed the mean preoperative ejection fraction (EF) was $56.63 \% \pm 11.85 \% ; 9 \%$ of patients presented an EF $<40 \%$ (Table2). Three patients were suffering from Hepatitis B and one case was Hepatitis C positive. One patient was hypothyroid and another was hyperthyroid. Data associated with types of operation and the number of mortalities is shown in Table3. Off-pump coronary artery bypass (32.27\%) was the most commonly performed surgery followed by mitral valve replacement (24.28\%). Sixty-five patients who died had undergone on-pump cardiac surgery with an average total cross-clamp time of $32.56 \pm 11.55$ minutes and an average total bypass time of $80.57 \pm 18.09$ minutes (Table4).

Most of the patients died in the first two weeks after surgery (Figure 1). Evaluation of death certificates of patients revealed irreversible cardiopulmonary arrest, in general, as the major cause of mortality. The specific reasons mentioned were arrhythmia like ventricular tachycardia and fibrillation, postoperative myocardial infarction, sepsis, multi-organ failure, systemic inflammatory response syndrome, acute respiratory distress syndrome, respiratory failure, etc.

Table 1. Baseline characteristics of the study population.

PTMC: Percutaneous transvenous mitral commissurotomy, IHD: Ischemic heart disease and MI: Myocardial infarction.

Table 2. Preoperative Echocardiography findings.

LVIDd: Left ventricular internal dimension in systole, LVIDs: Left ventricular internal dimension in diastole, FS: Fractional shortening, LVEF: Left ventricular ejection fraction and MAP: Mean Arterial Pressure.

Table 3. Types of surgical interventions.

OPCAB: Off-pump coronary artery bypass, CABG: Coronary artery bypass grafting, MVR: Mitral valve replacement, AVR: Aortic valve replacement, CMC: Closed mitral commissurotomy, ASD: Atrial septal defect and VSD: Ventricular septal defect.

Table 4. Details of cardiopulmonary bypass and postoperative inotropic support.

Figure 1. Distribution of the post-operative days of mortality.

World Health Organization International Coding of Diseases (WHO-ICD) is recommended for proper documentation of causes of mortality. Supplementary data are provided to show other results of the study like preoperative investigation findings (TableS1), ECG findings (TableS2), Chest X-ray findings (TableS3), and Spirometry findings (TableS4).

\section{Discussion}

Coronary artery disease (CAD) is increasing in the South Asian population, affecting the people at a relatively younger age with the severe and diffuse form of lesions. They have higher rates of CAD compared to other populations, more than two folds at any given level of conventional risk factors like obesity, hypertension, cigarette smoking, and high cholesterol. Myocardial infarction (MI) is seen in the South Asians 5 to 10 years earlier than other populations, and 5 to 10 folds higher rate of MI death occurs in those $<40$ years of age. Prevalence of new emerging risk factors like high levels of lipoprotein (A), triglycerides, fibrinogen, low levels of HDL is higher. Similarly, the prevalence of causes of glucose intolerance namely insulin resistance syndrome, diabetes, and central obesity are also increasing among young South Asians. This group of patients develops larger MI with greater myocardial damage and complications, the higher rate of clinical CAD events for a given degree of atherosclerosis, comparatively two-folds higher than the white population, and four-folds higher than the Chinese population [9] [10]. All these and the increasing prevalence of the conventional risk factors and high cholesterol where cholesterol levels similar to whites but higher than other Asian populations add to the disease burden [11]. 
In this systematic review of 100 cases of mortality after cardiac surgery, most of the risk factors and predictors of morbidity and mortality correlate well with the findings of this study. The data and indices of this study are of high quality and are used in national as well as international parameters to measure adverse events and risks in cardiac surgery. The study evaluated the comprehensive aspects usually considered as well as emerging risk factors like RDW and thyroid function tests were also evaluated. Cardiac surgery is relatively emerging as a safe surgery due to advances in preoperative, intraoperative as well as postoperative management. However, in the higher risk groups, a significant number of patients are still susceptible to prolonged morbidity and mortality [12] [13].

In this study, most of the patients belonged to the age group of $40-50(27 \%)$ or $50-60(26 \%)$, whereas in most literature octogenarians and septuagenarians were the most affected group [14]. The earlier age group possibly because the South Asian population has a higher risk compared to other racial groups of a major cardiac event in the early phase of the disease [15]. Postoperative mortality is highest in the first two weeks, which reflects the real burden of surgery. Coherently compared to other studies, this is considered as an independent predictor. This may be because the early postoperative period is metabolic and biochemical stress and most patients with cardiac disease cannot cope up with the surgical stress [16]. This study revealed that the mortality was much reduced after the third week $(\mathrm{f}=6 \%)$ suggesting that cardiac surgery patients are relatively stable after that period. Most of the patients had chronic illnesses like diabetes mellitus (f $=36 \%$ ), hypertension ( $\mathrm{f}=35 \%$ ) but in retrospect, they made up only $1 / 3 \mathrm{rd}$ of mortality cases, meaning mortality is influenced by many other factors. However, risk factors like hypertension, diabetes mellitus, etc. should be well controlled before scheduling the patients for elective cardiac surgery for a better outcome.

Similarly, BMI is considered a strong predictor of mortality [17], but in this study, most of the mortality was seen amongst normal BMI, hence, also suggesting mortality in post-cardiac surgery cases to be a concoction of multitude factors. An increased level of plasma RDW and CRP levels were found associated with the mortality of the patients. Lappé et al. found similar results in their study [18]. In this study, the bulk of the cases were CABG and valvular heart disease, namely mitral valve disease, and that was reflected in the numbers of mortality as well, ( $\mathrm{f}=30 \%, \mathrm{f}=25 \%$; respectively). During the study period, increased total cross-clamp time $(32.56 \pm 11.55 \mathrm{~min})$ was noticed in mitral valve disease with increased total bypass time $(80.57 \pm 18.09 \mathrm{~min})$ and inotrope time $(8.19 \pm 2.09$ days $)$, which may be due to being the initial phase of cardiac surgery, with a long learning curve and limited resources.

We recommend that more advance but fundamental facilities should be made available in cardiac centers like intraoperative transesophageal echocardiography, postoperative echocardiography, etc. to improve the outcome. Proper perioperative evaluation of risk factors and comorbidities should be carried out and wellcontrolled before the surgery. Preoperative respiratory exercise with incentive spirometry should be taught to the patients, if not contraindicated. Similarly, postoperative good respiratory care with proper respiratory physiotherapy and incentive spirometry is recommended to prevent postoperative respiratory complications like atelectasis, pneumonia, acute respiratory distress syndrome, etc. Transparent and well-managed data recording practice is of paramount importance in developing countries. More opportunities should be provided to the cardiac surgeons, nurses, and health care providers of the developing nations to improve the cardiac operative skills and care provided to the patients in such countries.

\section{Limitations}

The study is limited by its retrospective nature. This analysis was done with a small population of patients in a single-center of Bangladesh for a limited period and the sample only represents a small fraction of patients undergoing cardiac surgery. Medical records of the patients were paper-based which need to be computerized and properly recorded. The study is limited to simple statistical analysis because no comparative groups were considered to produce sophisticated statistical results explaining the association or causes of mortality after cardiac surgery.

6. Conclusion

Mortality after cardiac surgery is multifactorial. Regular analysis of the postoperative mortality after cardiac 
surgery should be done so that preventive measures may be focused. Many reliable causes for mortality may not truly represent the scenario of developing countries due to a lack of data. So, a large-scale prospective study with comparative groups is required to find out preventable measures of mortality after cardiac surgery in low-income countries.

Conflict of Interest

The authors have no conflicts of interest to declare. The preprint of the paper is available at Authorea (DOI: 10.22541/au.158981360.05697032).

References

1. Siregar, S., Groenwold, R.H., de Mol, B.A., Speekenbrink, R.G., Versteegh, M.I., Brandon Bravo Bruinsma, G.J., Bots, M.L., van der Graaf, Y. and van Herwerden, L.A. (2013) Evaluation of Cardiac Surgery Mortality Rates: 30-Day Mortality or Longer Follow-Up? European Journal of Cardio-Thoracic Surgery, 44, 875-883. https://doi.org/10.1093/ejcts/ezt119

2. Omar, R.Z., Ambler, G., Royston, P., Eliahoo, J. and Taylor, K.M. (2004) Cardiac Surgery Risk Modeling for Mortality: A Review of Current Practice and Suggestions for Improvement. The Annals of Thoracic Surgery, 77, 2232-2237. https://doi.org/10.1016/j.athoracsur.2003.10.032

3. Pons, J.M., Espinas, J.A., Borras, J.M., Moreno, V., Martin, I. and Granados, A. (1998) Cardiac Surgical Mortality: Comparison among Different Additive Risk-Scoring Models in a Multicenter Sample. Archives of Surgery, 133, 1053-1057. https://doi.org/10.1001/archsurg.133.10.1053

4. Kaplan, J.A. (2008) Essentials of Cardiac Anesthesia. Saunders, Philadelphia, 7.

5. Shannon, F.L., Fazzalari, F.L., Theurer, P.F., Bell, G.F., Sutcliffe, K.M. and Prager, R.L., Michigan Society of Thoracic and Cardiovascular Surgeons (2012) A Method to Evaluate Cardiac Surgery Mortality: Phase of Care Mortality Analysis. The Annals of Thoracic Surgery, 93, 36-43. https://doi.org/10.1016/ j. athoracsur.2011.07.057

6. Nowicki, E.R., Birkmeyer, N.J., Weintraub, R.W., Leavitt, B.J., Sanders, J.H., Dacey, L.J., Clough, R.A., Quinn, R.D., Charlesworth, D.C., Sisto, D.A. and Uhlig, P.N. (2004) Multivariable Prediction of InHospital Mortality Associated with Aortic and Mitral Valve Surgery in Northern New England. The Annals of Thoracic Surgery, 77, 1966-1977. https://doi.org/10.1016/j . athoracsur.2003.12.035

7. Kamal, Y.A., Al-Elwany, S., Ghoneim, A. and El-Minshawy, A. (2017) Traditional Predictors of In-Hospital Mortality after Coronary Artery Bypass Grafting: Current Status. Cardiothoracic and Vascular Science, 1, 1-5. https://doi.org/10.15761/CVS. 1000107

8. Nashef, S.A., Roques, F., Sharples, L.D., Nilsson, J., Smith, C., Goldstone, A.R. and Lockowandt, U. (2012) EuroSCORE II. European Journal of Cardio-Thoracic Surgery, 41, 734-745. https://doi.org/10. 1093/ejcts/ezs043

9. Rao, G.H.R. (2005) Coronary Artery Disease: Risk Factors, Pathophysiology and Prevention. Jaypee Medical Publishers, New Delhi, India, 21-57.

10. Ranjan, R., Joshi, D., Ullah, M.A., Bajracharya, P., Saha, S. and Adhikary, A.B. (2019) A 50-Year-Old Female, Diabetic Patient with Chest Pain and Dizziness. Bangabandhu Sheikh Mujib Medical University Journal, 12, 187-191. https://doi.org/10.3329/bsmmuj .v12i4. 44274

11. Yusuf, S., Hawken, S., Ôunpuu, S., Dans, T., Avezum, A., Lanas, F., McQueen, M., Budaj, A., Pais, P., Varigos, J. and Lisheng, L. (2004) Effect of Potentially Modifiable Risk Factors Associated with Myocardial Infarction in 52 Countries (the INTER- HEART Study): Case-Control Study. The lancet, 364, 937952. https://doi .org/10.1016/S0140-6736(04)17018-9

12. Hammermeister, K.E., Burchfiel, C., Johnson, R. and Grover, F.L. (1990) Identification of Patients at Greatest Risk for Developing Major Complications at Cardiac Surgery. Circulation, 82, IV380-389. PMID: 
13. Hossain, M.A., Vaidya, S., Acharya, M., Joshi, D., Sunny, S.A. and Khan, O.S. (2019) Atypical Location of an Atrial Myxoma: A Case Report. Mymensingh Medical Journal: MMJ, 28, 705-707. PMID: 31391449

14. Oskvig, R.M. (1999) Special Problems in the Elderly. Chest, 115, 158S-164S. https://doi.org/10. 1378/chest.115.suppl_2.158S

15. Gupta, M., Brister, S. and Verma, S. (2006) Is South Asian Ethnicity an Independent Cardiovascular Risk Factor? Canadian Journal of Cardiology, 22, 193-197. https://doi .org/10.1016/S0828-282X (06) 70895-9

16. Desborough, J.P. (2000) The Stress Response to Trauma and Surgery. British Journal of Anaesthesia, 85, 109-117. https://doi.org/10.1093/bja/85.1.109

17. Ri, M., Aikou, S. and Seto, Y. (2018) Obesity as a Surgical Risk Factor. Annals of Gastroenterological Surgery, 2, 13-21. https://doi.org/10.1002/ags3.12049

18. Lappé, J.M., Horne, B.D., Shah, S.H., May, H.T., Muhlestein, J.B., Lappé, D.L., Kfoury, A.G., Carlquist, J.F., Budge, D., Alharethi, R. and Bair, T.L. (2011) Red Cell Distribution Width, C-Reactive Protein, the Complete Blood Count, and Mortality in Patients with Coronary Disease and a Normal Comparison Population. Clinica Chimica Acta, 412, 2094-2099. https://doi.org/10.1016/j.cca.2011.07.018

Supplementary Data

Table S1. Preoperative investigation findings.

ESR: Erythrocyte Sedimentation Rate, WBC: White Blood Cell, N/L: Neutrophil/Lymphocyte, PCV: Packed Cell Volume, MCV: Mean Corpuscular Volume, MCH: Mean Corpuscular Hemoglobin, MCHC: Mean Corpuscular Hemoglobin Concentration, RDW-CV: Red Cell Distribution Width Coefficient of variance, RDW-SD: Red Cell Distribution Width Standard Deviation, MPV: Mean Platelet Volume, PDW: Platelet Distribution Width, RBS: Random Blood Sugar, FBS: Fasting Blood Sugar, 2HABF: Blood Sugar 2 hours after Breakfast, HbA1c: Glycosylated Hemoglobin A1c, CRP: C-reactive Protein, ALT: Alanine Aminotransferase, PT: Prothrombin Time, INR: International Normalized Ratio and APTT: Activated Partial Thromboplastin Time.

Table S2. Preoperative ECG findings.

Table S3. Preoperative chest X-ray findings.

Table S4. Preoperative spirometry findings. 